\title{
Establishment of human tumor xenografts in immunodeficient mice
}

\author{
Christopher L Morton \& Peter J Houghton
}

Department of Molecular Pharmacology, St. Jude Children's Research Hospital, Memphis, Tennessee 38105, USA. Correspondence should be addressed to P.J.H. (peter.houghton@stjude.org).

Published online 22 February 2007; doi:10.1038/nprot.2007.25

Heterotransplantation of human cancer cells or tumor biopsies into immunodeficient rodents (xenograft models) has, for the past two decades, constituted the major preclinical screen for the development of novel cancer therapeutics. Despite limitations, these models have identified clinically efficacious agents, and remain the 'workhorse' of the pharmaceutical industry. However, if therapeutic approaches to treating tumors according to their molecular characteristics are to be achieved, additional new models of human cancer will be required to represent the genetic diversity that exists within tumor histologies. This protocol details a method for establishing xenografts from primary solid-tumor isolates or cells grown in culture. The procedure relies on immunodeficient mice to provide a host for the establishment of human xenografts. The procedure can be completed in 1-2 $\mathrm{h}$ with results being obtained in 1-4 months.

\section{INTRODUCTION}

Animal models have been used as the front line in predicting efficacy and finding toxicities for cancer chemotherapeutic agents before entering the clinic. This has resulted in the development of many different animal models of malignant disease. The bulk of these models can be separated into two groups: first, grafts of tumor material (syngeneic or xenogeneic) into immunocompetent or immunodeficient animals, respectively; and second, genetically engineered mice that recapitulate a specific cancer genotype. Although these two groups both possess unique qualities, the usefulness of genetically engineered mice for identifying novel compounds that subsequently demonstrate significant clinical activity against the appropriate histology remains to be validated.

The discovery of nude athymic (nu/nu) mice that were T-cell deficient $^{1}$, and later B-cell-deficient and T-cell-deficient severe combined immunodeficient (scid/scid) mice ${ }^{2}$, allowed the routine and efficient transplantation and propagation of human tumor tissues (xenografts) in mice. These mouse strains allow established in vitro human cell lines to be propagated subcutaneously, reconstituting solid tumors. Human tumor tissue explants obtained from biopsy or autopsy can also be transplanted directly into these strains of mice.

The predictive value of these models has been debated extensively. In the National Cancer Institute (NCI) screening program, the activity of an agent in vitro did not predict for activity in either hollow-fiber assays in vivo or subcutaneous xenograft models, which in turn did not predict for activity against clinical cancer ${ }^{3}$. However, activity of an agent against breast cancer xenografts correlated with clinical activity for the agent against one or more cancers. Furthermore, activity in $30 \%$ of tumor models predicted for activity in some clinical cancer. In contrast to the NCI experience, retrospective analysis showed that xenograft models, but not allografts of murine tumors, can be useful for predicting the phase 2 clinical trial performance (at least for ovarian and non-small-cell lung cancers) when panels of tumors are used ${ }^{4}$. Other studies have found the predictive value of xenograft models to be variable ${ }^{5,6}$. For childhood cancers, xenograft models have been relatively accurate in identifying clinically active agents ${ }^{7}$ and effective drug combinations, particularly when differences in drug exposure between tested animals and humans are taken into account ${ }^{8}$.

However, the reason why the models failed to accurately predict activity in the appropriate clinical histology in the NCI program is of concern. In part, this might be a consequence of using continuous cell lines in vitro as the source of material for starting xenografts. In vitro culture for several years might select for clones that are no longer representative of the original tumor. For example, mutation of p53 or silencing of the gene encoding the DNA repair protein MGMT occurs relatively frequently in culture $^{9,10}$. Alternatively, as the culture of only a minority of tumor biopsies results in the generation of continuous cell lines, the process of in vitro culture might itself select for subsets of lines that are not representative of the disease per se.

Goldin and colleagues originally proposed using panels of xenografts to represent the 'clinical' disease ${ }^{11}$. However, with the continuing molecular-genetic subclassification of tumors within a histology it is clear that many tumor lines will be required to simulate the compound genetic diversity needed to model a clinical population with respect to, for example, estrogen receptor status or BRCA1 mutations in breast carcinoma. Similarly, modeling the dysregulation of the multiple membrane receptor kinase-signaling pathways - that is, endothelial growth factor (EGF), plateletderived growth factor receptor (PDGFR), insulin-like growth factor I (IGF-I), basic fibroblast growth factor (bFGF) and hepatocyte growth factor/scatter factor (HGF-SF) - reported for glioblastoma multiforme, or modeling subtypes of childhood sarcomas characterized by different chimeric transcription factors, will require the development of new xenografts.

For xenograft models to be valuable in the development of molecularly-targeted agents, careful molecular characterization is essential to their establishment and validation. Current genomewide molecular-profiling studies indicate the retention of molecular characteristics that define tumor type. The recent study by Whiteford and colleagues ${ }^{12}$ demonstrates that, by unsupervised clustering analysis of cDNA-expression profiles, xenografts derived from a histology can cluster accurately with their human 
counterparts. Similarly, direct comparison of patient tumor biopsy tissue with early-passage xenografts demonstrates high concordance in gene expression and even greater similarity in genomic alterations when tumors are propagated in mice. Historically, attempts have been made to use heterografts under the subrenal capsule as a method of predicting patient tumor chemosensitivity ${ }^{13}$. However, the low success of heterografting, and the relatively long time needed to establish tumor heterografts make this use of xenografts suboptimal. Rather, the value of panels of xenografts representing a particular histotype is to select novel agents that have a high response rate against that tumor type in patients, when administered to mice at doses that result in clinically achievable system exposures.

In addition to athymic nude mice, human tumor xenografts can be propagated in the subcutaneous space of scid/scid mice, non-obese (NOD)-scid mice and recombination-activating gene 2 (Rag2)-knockout mice. The protocol described covers the basic technique for establishing new tumors from patient biopsy, or for the serial transfer of mouse-propagated tumors. Although certain strains (NOD-scid) appear more receptive for growing leukemias ${ }^{14}$, our overall 'success' rate for establishing and serially propagating childhood solid tumors and brain tumors is high. For rhabdomyosarcoma and osteosarcoma, the success rates are $17 / 42$ (40\%) and 16/34 (47\%), respectively. For pediatric brain tumors, rhabdoid tumors of the CNS and kidney, and Wilms tumor (nephroblastoma), successful grafts were obtained from 49 of 156 samples (31\%), which included some that were found subsequently to be nonmalignant. Interestingly, the success rate with glioblastoma is low $(<20 \%)$, which might reflect the requirement for orthotopic heterotransplantation for these brain tumors. Thus, overall, the successful heterotransplantation rate is higher than that reported for adult carcinomas, particularly those of the prostate and breast. This might reflect a difference in the biology of pediatric cancers, or the difficulty in heterografting hormone-dependent tumors. It is, however, important to realize that the chemosensitivity of human tumors heterografted into different strains might differ, suggesting that the genetic background of the host mouse might be a factor in determining tumor responsiveness ${ }^{15}$.

In this protocol, we present methods for establishing a tumor xenograft in mice from tumor tissue or cells from cell culture.

\section{MATERIALS}

REAGENTS

- CB17SC-M-F scid ${ }^{-/-}$mice, 6-10 weeks old (Taconic Farms, mod. no. CB17SC-M) ! CAUTION All mice must be maintained under barrier conditions; experiments must be conducted according to the relevant national regulations, using protocols and conditions approved by the animal care and use committee of the institution where the experiment is performed

- Medium 199 with Earle's salts, L-glutamine and sodium bicarbonate

(Invitrogen, cat. no. 11150-059)

- Penicillin/streptomycin solution 100X (Sigma, cat. no. P4333)

-A-33 disinfectant (Ecolab Professional Products, cat. no. 61122362)

-3 M Vetbond tissue adhesive (Webster Veterinary Supply, cat. no. 480200) $\cdot 70 \%(\mathrm{vol} / \mathrm{vol})$ ethanol

- Isoflurane, USP (Baxter)

- Gauze squares

EQUIPMENT

- Sterile instruments (scalpel, scissors and forceps)

- Sterile Petri dishes

- Anesthesia machine/chamber (VetEquip)

- Class 2 (Type A/B3) biological safety cabinet (Baker)

- Coulter counter (Beckman Coulter) or hemocytometer

- 1 cc tuberculin (TB) syringe

-21 G needle

\section{PROCEDURE}

Preparation

1) Prepare the work area by disinfecting all hood surfaces with A-33.

ICAUTION All in vitro and in vivo procedures should be undertaken in a class 2 biological safety cabinet to ensure sterility.

I CAUTION All procedures used for handling human tissues should assume potential contamination of tissue with human pathogens.

2| Follow option A if establishing new xenograft tumors from patient tissues, or option B if establishing xenografts from established cell lines.

(A) Preparing tissue from new xenograft tumors from patient tissues for transplant.

(i) Prepare media working stock by adding penicillin/streptomycin solution in a 1:100 dilution to Medium 199.

(ii) Place the anesthesia chamber in the cabinet, and set the oxygen/isoflurane mixture according to the manufacturer's instructions.

(iii) Transfer the tumor to a sterile Petri dish containing Medium 199 plus penicillin/streptomycin. It is important that enough media is present to bathe the tumor. This ensures the viability of the transplant material.

(iv) Cut the tumor into pieces about the size of a pencil eraser $(5 \times 5 \times 5 \mathrm{~mm})$. Care should be taken to make the pieces as uniform as possible. Ensure that any necrotic tissue is removed and is not used for transplant. Necrotic tissue can vary in appearance in different tumor types, but is generally characterized as dark liquefied tissue at the center of a large tumor.

(B) Harvesting cells from culture.

(i) Harvest cells grown in monolayer culture during the exponential growth phase using trypsin or appropriate enzymes for the specific cell line. Suspend cells in medium plus serum.

I CAUTION Cells should be tested to ensure that they are not contaminated with viruses that would be harmful to immunodeficient mice. This can be accomplished using the traditional murine antibody production (MAP) test or one of the more sensitive PCR-based tests, such as the University of Missouri Research Animal Diagnostic Laboratory (RADIL) infectious microbe PCR amplification test (IMPACT). 
(ii) Quantitate the number of cells present using a Coulter counter or a hemocytometer.

(iii) Centrifuge cells at $225 \mathrm{~g}$ and $20^{\circ} \mathrm{C}$ for $5 \mathrm{~min}$. Remove the medium, and re-suspend the cells in medium without serum at a concentration of $1 \times 10^{8}$ cells per $\mathrm{ml}$ of medium.

\section{Transplant}

3| Place the recipient CB17SC-M-F scid ${ }^{-/-}$mice in the anesthesia chamber following the manufacturer's recommended settings. I CAUTION Appropriate protective measures should be taken to ensure that workers are not exposed to waste anesthetic gases using suitable activated-charcoal capture or exhaust venting.

4| After a mouse reaches sufficient anesthetic depth, remove it from the chamber and place it (ventral side down) in a properly sized nose cone, configured according to the manufacturer's recommended settings, with the dorsal side facing upwards. To confirm anesthetic depth, pinch the rear foot lightly. If no kicking response is present, continue with the procedure.

5| Follow option A (surgery) if establishing new xenograft tumors from patient tissues, or option B (injection of cells) if establishing xenografts from established cell lines.

\section{(A) Transplant of tumor tissue.}

(i) Using a gauze square saturated with $70 \%(\mathrm{vol} / \mathrm{vol})$ ethanol, wipe the area from the mid-spine to the base of the tail to prepare for the incision.

(ii) Make a small, horizontal incision of $5 \mathrm{~mm}$ in length, $10 \mathrm{~mm}$ above the base of the tail, using small surgical scissors.

(iii) Insert the tip of the scissors into the incision, directly over the flank, and open the scissors to introduce a pocket in the subcutaneous space.

(iv) Insert one individual piece of tumor into the pocket created using forceps.

(v) While the pocket is still open, place one drop of $100 \mathrm{X}$ penicillin/streptomycin solution into the opening.

(vi) Close the incision site with one drop of Vetbond tissue adhesive. Hold the tissue together for 3-5 $\mathrm{s}$ with forceps to allow drying.

(B) Injection of cell suspension.

(i) Agitate the cell suspension to prevent the cells from settling, and withdraw from the sterile tube into a 1-cc TB syringe with the needle removed.

(ii) Lift the skin of the mouse to separate it from the underlying muscle and inject, with a $21 \mathrm{G}$ needle, $0.1 \mathrm{ml}$ of the cell suspension $\left(1 \times 10^{7}\right.$ cells) subcutaneously.

6| Place the animal in a clean cage and observe for 10-15 min to ensure recovery from the anesthetic.

\section{ANTICIPATED RESULTS}

For the first week following implantation, a small bump will be visible where the tumor was inserted and this will then disappear. At 1-4 months (and occasionally up to $1.5 \mathrm{yr}$ ) following implantation, a tumor will begin to appear at the site of implantation. This visualization is made easier by trimming the hair from the haunch using animal trimmers. When the tumor reaches $\sim 1.5 \mathrm{~cm}$ in diameter, it can be excised, dissected into $5 \mathrm{~mm}$ cubes and transplanted into additional mice using the same procedure. It is important to characterize the tumor at this point, to ensure that it is derived from the starting material. The most accurate method for doing this is to harvest genomic DNA from both the primary sample and the established xenograft tumor. These separate DNA samples can be analyzed by single-nucleotide polymorphism (SNP) array to determine whether they arise from the same sample. Once tumor integrity has been confirmed, the xenograft can be transplanted into additional mice for chemosensitivity testing. Following transplantation, tumors should be allowed to grow to $200-500 \mathrm{~mm}^{3}$ before initiation of treatment.

COMPETING INTERESTS STATEMENT The authors declare that they have no competing financial interests.

Published online at http://www.natureprotocols.com Reprints and permissions information is available online at http://npg.nature.com/ reprintsandpermissions

1. Flanagan, S.P.'Nude', a new hairless gene with pleiotropic effects in the mouse. Genet. Res. 8, 295-309 (1966).

2. Bosma, M.J. \& Carroll, A.M. The SCID mouse mutant: definition, characterization, and potential uses. Annu. Rev. Immunol. 9, 323-350 (1991).

3. Johnson, J.I. et al. Relationships between drug activity in NCI preclinical in vitro and in vivo models and early clinical trials. Br. J. Cancer 84, 1424-1431 (2001).

4. Voskoglou-Nomikos, T., Pater, J.L. \& Seymour, L. Clinical predictive value of the in vitro cell line, human xenograft, and mouse allograft preclinical cancer models. Clin. Cancer Res. 9, 4227-4239 (2003).
5. Bellet, R.E., Danna, V., Mastrangelo, M.J. \& Berd, D. Evaluation of a "nude" mousehuman tumor panel as a predictive secondary screen for cancer chemotherapeutic agents. J. Natl. Cancer Inst. 63, 1185-1188 (1979).

6. Boven, E., Winograd, B., Fodstad, 0., Lobbezoo, M.W. \& Pinedo, H.M. Preclinical phase II studies in human tumor lines: a European multicenter study. Eur. J. Cancer Clin. Oncol. 24, 567-573 (1988).

7. Houghton, P.J. et al. The pediatric preclinical testing program: description of models and early testing results. Pediatr. Blood Cancer 2007/D0I:10.1002/pbc 21059.

8. Peterson, J.K. \& Houghton, P.J. Integrating pharmacology and in vivo cancer models in preclinical and clinical drug development. Eur. J. Cancer 40, 837-844 (2004).

9. Harris, L.C. et al. Changes in 06-methylguanine-DNA methyltransferase expression during immortalization of cloned human fibroblasts. Carcinogenesis 17, 219-224 (1996).

10. Taylor, A.C. et al. P53 mutation and MDM2 amplification frequency in pediatric rhabdomyosarcoma tumors and cell lines. Med. Pediatr. Oncol. 35, 96-103 (2000). 
PROTOCOL

11. Goldin, A. et al. Current results of the screening program at the Division of Cancer Treatment, National Cancer Institute. Eur. J. Cancer 17, 129-142 (1981).

12. Whiteford, C. et al. Credentialing preclinical pediatric xenograft models using gene expression and tissue microarray analysis. Cancer Res. 67, 32-40 (2007).

13. Bogden, A.E., Cobb, W.R. \& LePage, D.J. The 6-day subrenal capsule assay (SRCA): its criticism, biology and review of assay/clinical correlations. Prog. Clin. Biol. Res. 276, 139-204 (1988).
14. Lock, R.B. et al. The nonobese diabetic/severe combined immunodeficient (NOD/ SCID) mouse model of childhood acute lymphoblastic leukemia reveals intrinsic differences in biologic characteristics at diagnosis and relapse. Blood 99, 4100-4108 (2002).

15. Yoshimura, M., Endo, S., Hioki, K., Ueyama, Y. \& Ohnishi, Y. Chemotherapeutic profiles of human tumors implanted in SCID mice showing appreciable inconsistencies with those in nude mice. Exp. Anim. 46, 153-156 (1997). 